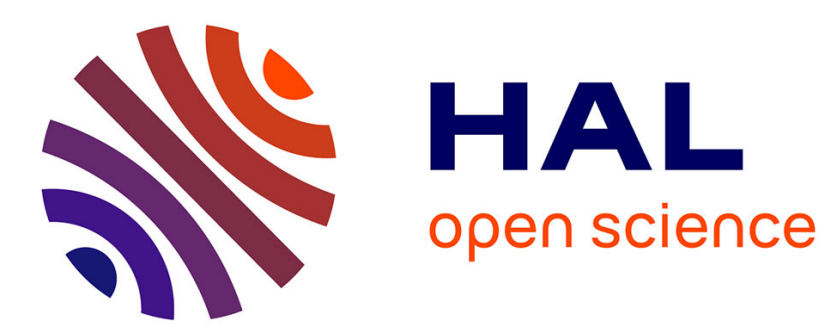

\title{
Les mouvements de " la transition " ou l'importance de la complémentarité
}

\author{
Fabrice Flipo
}

\section{To cite this version:}

Fabrice Flipo. Les mouvements de " la transition " ou l'importance de la complémentarité. Mouvements: des idées et des luttes, 2013, La transition, une utopie concrète?, 75, pp.99-109. 10.3917/mouv.075.0099 . hal-01140715

\section{HAL Id: hal-01140715 https://hal.science/hal-01140715}

Submitted on 9 Apr 2015

HAL is a multi-disciplinary open access archive for the deposit and dissemination of scientific research documents, whether they are published or not. The documents may come from teaching and research institutions in France or abroad, or from public or private research centers.
L'archive ouverte pluridisciplinaire HAL, est destinée au dépôt et à la diffusion de documents scientifiques de niveau recherche, publiés ou non, émanant des établissements d'enseignement et de recherche français ou étrangers, des laboratoires publics ou privés. 


\section{Les mouvements de « la transition » ou l'importance de la complémentarité}

Flipo F., Les mouvements de la transition ou l'importance de la complémentarité, Mouvements, nº75, 2013 / 3, pp. 99109.

Fabrice Flipo

Comment interpréter le mode d'action des mouvements se réclamant de « la transition »? Il n'y a pas de théorie unifiée en la matière. Les critiques venant de la science politique sont connues : ces mouvements sont trop petits, ils perdent leur temps à réinventer chacun dans leur coin ce que d'autres ont déjà accompli ailleurs, ils devraient s'organiser et se fédérer pour trouver un « débouché politique » etc. Pourtant chacun semble bien avoir choisi sa stratégie en toute conscience des enjeux, et en ayant en tête toutes ces objections. Autrement dit les différentes initiatives, tout en reconnaissant leur caractère limité, semblent aussi se vivre en partie sur le mode de la complémentarité ou encore du contraste (ne parler que de « contre-pouvoir »serait réducteur), se posant la question d'une unification mais se demandant aussi s'il est temps ou possible de le faire, cela parce que chacune d'entre elles répond de fait à des situations particulières, en partie nonreproductibles, difficiles à généraliser, impliquant, pour s'étendre, une grande ouverture aux situations des autres, afin de les reconfigurer, pour les rapprocher, les fusionner, de proche en proche. Ces différences peuvent tenir à des éléments objectifs (ici une zone urbaine, là une zone rurale, ailleurs encore c'est une zone française, à la différence d'une zone catalane etc.) ou subjectifs (histoire locale, caractère fédérateur et dialectisant ou non de telle ou telle étiquette politique etc.). Les principes généraux restent les mêmes (engagement, participation etc.) mais les formes concrètes varient en fonction des affinités et des sensibilités (l'énergie, l'agriculture, les modalités non-violentes de résolution des différends, l'engagement en politique, le contre-pouvoir etc.), des compétences apportées, de la configuration locale etc. Une lecture qui partirait d'emblée «d'en haut», avec des catégories «déjà faites", ancrées dans l'échelle nationale voire internationale produirait un effet de blocage, en voulant surimposer une lecture qui ne serait pas celle de toutes et de tous, et se trouverait ainsi en décalage avec les réalités vécues et pratiquées.

La plupart des théories politiques cherchent à proposer une conception unifiée de l'analyse et de l'action, sur le modèle de la lutte des classes, au moins implicitement, comme si l'unité était faite et non à faire. Or depuis près de cinq décennies, c'est à un phénomène inverse que nous assistons : une prolifération de théories de « la différence », correspondant à un ensemble divers de mouvements (genre, écologie, droit des animaux, migrations, culture etc.). Deux grands courants critiques s'affrontent : le parti «culturaliste » (minorités, discriminations, « care » etc.) et ceux pour qui la lutte économique reste la priorité. Dans les années 60, alors que le marxisme classique était en lente déliquescence, sous les coups de boutoir conjugués des désillusions $\mathrm{du}$ « socialisme réel » et de l'embourgeoisement de la classe ouvrière, ainsi que de l'échec du marxisme face au fascisme, un grand nombre de théoriciens se sont tournés vers les études de terrain, la psychanalyse et la psychologie sociale pour essayer de fonder une nouvelle «dialectique» des sociétés humaines. Reich, Marcuse, Adorno, Deleuze \& Guattari, Sartre, Gorz, Althusser, même, Castoriadis et bien d'autres ont entrepris, à la suite notamment des leçons de Kojève sur Hegel, de « revenir » au moment subjectif, pour chercher à donner un fondement au marxisme, qui semblait plus n'en avoir aucun. Sur quelle subjectivité collective appuyer la critique de l'ordre existant? Sur quels besoins non satisfaits ? Sur quelle conception de la richesse ? Le marxisme classique, mécaniste, abordant tout comportement de la classe ouvrière qui ne serait pas conforme à la théorie comme un problème de « conscientisation », n'était plus convaicant. 
Nous proposons ici quelques éléments théoriques trop rarement mentionnés, qui nous semblent pouvoir éclairer quelques aspects de la stratégie des mouvements évoqués dans ce numéro. Il resterait à les tester empiriquement de manière plus étroite.

\section{Facticité, groupes et collectifs}

Un point de rencontre de tous ces mouvements est l'insistance sur le faire, l'action dans sa dimension matérielle et concrète : poser des actes, dans l'espace public. Cette caractéristique trouve des échos chez Sartre qui, dans L'Être et le Néant (1943), souligne que la liberté est «facticité ». Si nous n'étions que de purs esprits, nous serions sans prise sur le réel. La liberté est originellement rapport au donné, qui se donne comme pouvant être changé. Que nous soyons facticité implique d'être toujours-déjà sous le regard des autres : ma matérialité et son évolution témoigne de manière visible de ce que je suis et de la liberté que j'incarne. Nous sommes ce corps-là, dans ce lieu-là, avec cette famille-là etc. que nous n'avons pas complètement choisi, ce donné en constante évolution, que nous pouvons réorienter. Nous « transcendons » le donné, c'est encore un fait matériel : nous sommes «jetés » dans un monde dont l'ordre est largement contingent, et peut donc être changé. Dans ce contexte, exister, c'est se situer $^{1}$, évaluer les points d'appuis qui permettent d'aller dans le sens voulu. Et se situer, c'est définir dans quelle mesure je suis aliéné ou pas. Le monde est habité par d'autres que moi, qui sont aussi des forces matérielles et des transcendances, qui échappent toujours partiellement à mon investigation, comme je leur échappe aussi, réciproquement. Je ne connais ce qu'ils pensent et ce à quoi ils tiennent que par leur manifestation empirique, matérielle - soit par leurs dires, soit par leurs actions.

Cherchant à interpréter la révolution française ${ }^{2}$, Sartre propose de distinguer deux modalités de l'êtreensemble humain, qu'il appelle des « ensembles pratiques » : le groupe et le collectif. Dans le collectif l'état de chacun est celui de la « sérialité » : les individus coexistent comme isolés, extérieurs les uns aux autres, et c'est cette relation d'extériorité mutuelle qui confère sa structure au collectif. Chacun est pure matérialité, inertie pour les autres. Ainsi par exemple une file de bus : chacun dispose ses actions en fonction de la configuration du milieu, sans avoir besoin de parler. Quel que soit l'individu qui se présente pour attendre le bus, les comportements sont à peu près les mêmes. Même chose dans une usine. Les individus se comportent comme des « séries » au sens industriel du terme : un lot de pièces matérielles aux formes et aux propriétés très similaires. C'est par pure contingence qu'un individu donné ne pourra pas prendre le bus et devra attendre le bus suivant s'il n'y a pas assez de place pour tout le monde. La « classe » sociale, dit Sartre, est une série de ce genre. Tout ouvrier est face à une machine. Cette condition sérielle est à l'origine de la « conscience de classe ». Et si les séries se défont, comme on a pu le voir avec la montée du tertiaire, et l'unité pratique se dissout, ou du moins s'affaiblit. D'autres séries se forment, sur les retraites ou contre tel ou tel traité européen. Dans le collectif, la réciprocité est pseudo-réciprocité : il n'y a pas de concertation. Ce qui fait l'unité de l'ensemble social c'est la structure similaire des corps et des attentes, c'est un même rapport dialectique avec une situation. Le collectif est régi par une objectivité commune : chacun fait ce que fait l'autre. Les possibilités sont rigides et déterminées. Le nombre d'individus membres d'un collectif est indéfini : peu importe qui en est et combien ils sont, sauf si le nombre pose un problème matériel - par

1 Sartre J.-P., L'Être et le Néant, Paris, TEL-Gallimard, 1976, Éd. Orig. 1943, p. 348.

2 Sartre J.-P., Critique de la raison dialectique - 2 tomes, Paris, Gallimard, 1985, Ed. Orig.1960 (tome 1), 1985 (tome 2). 
exemple le nombre de postes de travail aux machines en rapport avec la taille du marché du travail détermine «l'armée de réserve » industrielle évoquée par Marx. C'est l'objet qui détermine la praxis ; il trace un destin. L'autre modalité des relations humaines est le groupe, qui «se définit par son entreprise et par ce mouvement constant d'intégration qui vise à en faire une praxis pure en tentant de supprimer en lui toutes les formes d'inertie $»^{3}$. Le groupe surgit du collectif lors d'un événement, d'un danger, d'une revendication qui fait sortir les individus de leur comportement sériel. L'événement crée en chacun une structure d'objectivité unifiante, au terme d'une « effervescence » fondée sur une dynamique d'imitation. Sartre prend l'exemple de Paris encerclée par le Roi pendant la révolution : celui qui voit courir court. Imiter est encore un comportement inerte; mais quand les citoyens commencent à se saisir des armes alors des groupes émergent : on se parle, on s'organise. Le groupe est alors « en fusion », tout le monde régule tout le monde, les messages circulent vite et personne ne se trouve au-dessus des autres. Chacun est dans une relation réciproque avec les autres. Chacun dissout activement son être sériel, utilise son inertie, sa force pour la mettre au service du projet commun. Chacun est être-commun. « Cette structure nouvelle et capitale de la réciprocité médiée se caractérise en ceci : je me vois venir au groupe en lui », il est mon objectivité vécue. La réciprocité a quatre caractéristiques : l'autre est moyen dans le même degré que je suis moyen moimême; je reconnais l'autre comme praxis c'est-à-dire totalisation en cours en même temps que je l'intègre à mon projet totalisateur; je reconnais son mouvement mes fins deviennent les siennes; je me découvre comme moyen instrument de ses fins aussi bien que l'inverse.

Le groupe peut aussi être groupe organisé, passer de l'un à l'autre ou inversement. La différence est que dans un groupe organisé, les fonctions sont différenciées, tout en servant encore la finalité commune. Sartre prend l'exemple d'une équipe de football : "chacun se fait dépendant de l'Autre en tant qu'ils sont tous deux les mêmes. La médiation des fonctions, c'est la praxis commune $»^{4}$. La réciprocité inerte est inertie réciproque. Chaque individu est essentiel, dans le groupe : si l'un d'entre eux vient à manquer c'est tout le groupe qui se fige. L'effet matériel sur le monde est fonction du groupe et non de l'individu. Le groupe donne ainsi une apparence trompeuse d'organicité. Mais le groupe peut s'institutionnaliser, c'est-à-dire redevenir un collectif, sériel. Ici l'individu devient inessentiel ; de tiers régulateur, exprimant sa liberté, il est devenu tiers réglé, devenant autre que lui-même, aliéné. L'institution se maintient dans la série par pure inertie, sans aucune justification: "c'est comme ça $»^{5}$. Tous les conservatismes voient dans ce destin la garantie d'un ordre social, et ne forment des groupes que pour le maintenir.

Dans les mouvements de « la transition », la question de la souveraineté est omniprésente : comment ne pas subir son destin? En termes sartriens : comment sortir d'un comportement sériel, dont la direction nous paraît néfaste ? Comment dissoudre la nécessité dans laquelle nous nous trouvons, obligés que nous sommes de nous servir d'une automobile pour nous déplacer, ou d'aller au supermarché pour consommer ? La souveraineté est un fait, dit Sartre, elle ne réclame donc aucun fondement. La souveraineté « est simplement le rapport d'intériorité univoque de l'individu comme praxis au champ objectif qu'il organise et dépasse vers sa propre fin $»^{6}$. L'erreur est de prendre l'état de massification («peuple», « nation » etc.) à titre de point de départ, de faire comme si ces entités étaient un groupe, un résultat voulu et désiré, fruit d'une réciprocité, alors qu'il peut n'être qu'un agrégat statistique de situations subies. L'erreur est aussi de ne pas s'ancrer dans une analyse soigneuse des possibilités qui s'offrent réellement aux individus, compte-tenu de leur isolement.

3 Sartre J.-P., Op. Cit., 1960, p. 363

$4 \quad$ Ibid., p. 568

$5 \quad$ Ibid., p. 721

$6 \quad$ Ibid., p. 695 
L'exemple de l'entretien avec Vincent Gerber le montre, dans ce numéro : de la « voie longue » pour changer le monde à la « voie courte », les étapes sont nombreuses, dénier leur existence rend incompréhensible la stratégie des acteurs, et revient à se demander pourquoi ils ne passent pas à travers les murs sans ouvrir de porte.

Quels sont les obstacles pratiques sur la voie d'un changement de situation ? Les partis se présentent naturellement comme la solution la plus évidente, mais ils oublient que l'État n'est pas tout-puissant : il n'est que quasi-souverain, il ne possède pas la part de souveraineté que détient chaque individu, envers et contre tout, et que seule l'élimination physique peut faire disparaître. Le quasi-souverain ne peut jouer que sur les séries existantes, et sur la formation de groupes dont il accepte qu'ils lui échappent. L'exemple de la revendication écologiste est une parfaite illustration de cette situation : il n'y a aucune configuration partidaire sous laquelle des mesures rapides pourraient être obtenues, pour de multiples raisons. Les militants qui deviennent ministres constatent bien souvent qu'ils ne peuvent « rien faire » ${ }^{7}$. Les observateurs qui concluent au « narcissisme » d'un mouvement tel que les « Indignés » s'en tiennent à donner des leçons, sans se salir les mains. Pour paraphraser ce que disait Marx à propos du socialisme utopique, ils substituent l'ingéniosité de leurs analyses à la difficulté des obstacles concrets. Il ne suffit pas de voter des lois ou des règlements, le changement social ne peut se produire de manière massive que si les sérialités sont là, comme c'était encore le cas de la lutte des classes, réduite aux revendications de revenus et de conditions de travail : tout le monde « travaille » et se retrouve devant les mêmes obstacles.

Si les indignés ne s'organisent pas en collectif, doté d'une direction, c'est peut-être parce qu'ils n'expriment qu'une sérialité, une communauté de situation, qui génère "l'indignation », mais ne trouve pas de débouché de masse (« politique ») clair, tout simplement parce que l'agrégat n'existe pas (encore). Sans un tel levier, un parti politique ou un gouvernement n'a guère de pouvoir. Les majorités sont toujours sérielles, elles ne valent pas soutien à une politique, mais peut-être seulement dispersion. On ne le sait que par l'épreuve des faits. Le quasi-souveraineté occupe toutefois cette position centrale qui le met au-dessus de la récurrence : il est le seul à parler à tous en même temps. Effet de retour, cependant : il est lui aussi pétrifié par les masses et leur sérialité. Il ne peut pas provoquer la fusion des séries à la place des individus eux-mêmes. « La limite du pouvoir réel de l'État le plus dictatorial, c'est qu'il ne peut créer de groupe en dehors de lui-même ${ }^{8}$. Un tel pouvoir finit inévitablement par se figer, car il engendre de la méfiance, donc de l'inertie, puisqu'il détruit la réciprocité sans laquelle il n'y a pas de changement possible, car pas de fusion des séries. L'État n'est légitime que par défaut, parce qu'aucun groupe ne le met en cause, soit parce qu'il ne le souhaite pas (les séries sont «satisfaisantes »), soit parce qu'il n’y parvient pas : le souverain empêche activement tout regroupement hors de lui-même, et même en son sein, par épurations successives - ainsi Staline agonise-t-il pendant deux jours, délaissé de tous, même de ses médecins, qui craignent de prendre une initiative erronée, conduisant au Goulag. Sa crainte est toujours de voir des groupes se former contre lui, pour prendre sa place. C'est la structure fondamentale de l'obéissance.

\section{Moscovici et « l'influence » sociale}

Les analyses qui se fondent sur des mouvements de masse tels que le mouvement ouvrier ont donc pour caractéristique implicite de se consacrer à l'étude de séries bien établies, et non de les construire. D'où ce constat de Marx, pour qui le mouvement ouvrier est «un mouvement spontané de l'immense majorité, au profit de l'immense majorité »". Au contraire les mouvements « de la transition » ont en commun d'être

7 Poujade R., Le ministère de l'impossible, Paris, Calmann-Lévy, 1975 ; Lepage C., On ne peut rien faire, Madame la ministre : chronique d'un État impuissant, Paris, Albin Michel, 1998.

8 Ibid., p. 744

9 Marx K., Le Manifeste du Parti Communiste, 10/18, 1962, Traduction Francis Brière, Éd. Orig. 1847, p. 34 
minoritaires, et plus proches de la structure du groupe sartrien, avec le souci d'une forte autodétermination interne. Comme le montre Moscovici, à la suite de Sartre, les modalités d'action et d'influence de tels mouvements sont radicalement différentes : « il y a des époques majoritaires, où tout semble dépendre de la volonté du plus grand nombre, et des époques minoritaires, où l'obstination de quelques individus, de quelques groupes restreints, paraît suffire à créer l'événement, et à décider du cours des choses $»^{10}$.

« Minorité » et « majorité » doivent naturellement s'entendre sous l'angle de rapports réciproques, car il n'y a de minorité que dans le rapport avec une majorité : «ce n'est pas le nombre, ce sont les rapports intérieurs au nombre $»^{11}$, c'est-à-dire le rapport de la majorité avec la minorité. Les théories politiques disponibles n'ont-elles pas tendance à ne s'intéresser qu'aux stratégies majoritaires, à l'instar du marxisme ? Dans ce cas les séries sont claires (lutte des classes) et les revendications sont manifestement partagées. Autre chose est de changer une manière d'utiliser l'énergie, où ce qui est en jeu est la mise en place de filières industrielles, d'activités nouvelles etc. ce qui, s'attaquant à des inerties de grande ampleur, prend nécessairement du temps, et passe par un grand nombre de « fusions » successives. La stratégie minoritaire est très différente de la stratégie majoritaire, fondée sur la masse. Elle a beaucoup plus recours au spectacle. Il faut qu'elle parvienne à se faire entendre de la majorité, dont les convictions sont souvent molles, pour réussir à la faire partiellement « fondre », et générer de nouvelles séries.

Serge Moscovici, l'un des fondateurs du mouvement écologiste, rangé par Jean Jacob parmi les « naturalistes actifs $»^{12}$, est l'un de ceux qui a travaillé sur les modalités d'influence d'une minorité sur la minorité. Ce qu'il indique «parle» d'ailleurs aux acteurs concernés (cf. notamment l'interview de Colibri). À la lumière d'expériences dont nous ne donnons pas le détail ici, Moscovici estime que toute personne est source d'influence autant que réceptrice, et que l'évolution se fait au moyen de création active d'incertitude, notamment au moyen du conflit. Ainsi la minorité arrive à faire douter la majorité de l'interprétation de sa situation, et amorcer de nouveaux cheminements possibles. La majorité est « sérielle », pour parler comme Sartre : elle n'adhère pas forcément au résultat collectif qu'elle produit. Elle ne s'y engage que parce qu'elle n'a pas d'autres possibilité immédiate, qu'elle ne peut créer un "groupe». Si elle veut provoquer un changement social de grande ampleur, la minorité se trouve dans une situation de la poule et de l'œuf : il n'y a pas de mouvement de masse parce qu'il n'y a pas de mouvement de masse. Il n'y a pas de masse parce qu'il n'y a pas de série, la dispersion est donc subie. Il n'y a pas de possibilité de sortir de cette situation sans partir de cette dispersion et l'agir, en tant qu'elle est une étape incontournable.

Dans cette situation la norme permettant la plus grande influence n'est pas l'objectivité (les « lois dialectiques de l'histoire», les «lois économiques» etc.) mais l'originalité, car elle respecte la transcendance de l'individu, elle fait appel à sa réalité privée, à sa volonté, ses désirs, sans l'enfermer par avance dans un résultat collectif, sans sauter par-dessus l'histoire. La norme d'originalité implique évidemment, du point de vue du groupe qui veut obtenir l'influence, de renoncer à produire des résultats totalement prévisibles, et donc de n'avoir pas de «théorie». Ceci peut expliquer pourquoi les mouvements de la transition ne souhaitent ni ne peuvent partager un même référentiel, un même mot d'ordre (« transition », mais aussi « décroissance », «buen vivir » etc.) ni même se regrouper pour réclamer " un programme commun ». Ce n'est pas faute de s'intéresser « au politique » : c'est constater que ce politique, si par là on désigne le lieu de changements « de masse », n'existe pas encore.

10 Moscovici S., Psychologie des minorités actives, Paris, PUF, 1996. Éd. Orig. 1979, p. 11

11 Deleuze G. \& F. Guattari, Capitalisme et schizophrénie, Tome 2. Mille plateaux, Paris, Éditions de Minuit, 1980, p. 587

12 Jacob J., Histoire de l'écologie politique, Paris, Albin Michel, 1999. 
Serge Moscovici, cherchant à déterminer quels sont les facteurs qui sont décisifs, dans une tentative d'influence d'une minorité sur une majorité, arrive à la conclusion que c'est le "style de comportement», qui renvoie à "l'organisation des comportements et des opinions, au déroulement et à l'intensité de leur expression, bref, à la "rhétorique " $d u$ comportement et de l'opinion ${ }^{13}$. Mais quels sont ces comportements ? Comment les organiser pour qu'ils contribuent au succès ? L'auteur en identifie cinq types : l'investissement (par exemple, le militantisme), l'autonomie (montrer qu'on est capable d'agir selon ses propres lois), la consistance (démontrer de la conséquence dans ses démarches, indice de conviction et de cohérence), la rigidité (ou inaptitude au compromis, autrement dit la " radicalité ») et l'équité (aptitude au compromis et à l'ouverture, au contraire). Chacun des cinq se trouve caractériser une attitude qui permet d'obtenir de l'influence. C'est un résultat empirique, ainsi par exemple Soljenitsyne, que Moscovici a beaucoup étudié. Ce contestataire n'a pas eu d'influence directe sur le gouvernement, en formant un parti, mais au travers d'actes symboliques, tels que refuser un poste important ou publier des textes interdits. Aucun des cinq n'est plus « efficace » que les autres : la réussite découle de l'adaptation du comportement à la situation. Être rigide à un moment où il s'agit de négocier peut conduire à réduire l'influence qu'on aurait pu avoir ; être systématiquement « radical» dans tous les domaines finit ainsi par couper les individus qui adoptent cette posture du reste de la société. Inversement négocier à tout bout de champ finit par détruire ce que la minorité a à porter en propre, et réduire à néant son influence. Trop de consistance en toutes circonstances finit par tuer l'originalité, car être « consistant» signifie conserver un ensemble de buts, pardelà les variations qui sont tolérées et même encouragées par la norme d'originalité. Il y a donc bien des bornes à l'originalité : ce qui fait litige reste commun, par-delà la diversité des manifestions et des actions. Et c'est pour cette raison que le profane, s'intéressant à ces mouvements sociaux que l'on regroupe ici un peu arbitrairement sous le mot de «transition », ne peut manquer de remarquer une certaine parenté d'ensemble, quelle que soit la bannière sous laquelle tel ou tel projet est mis en œuvre.

La majorité est « conformiste », ce qui revient à dire qu'elle est « sérielle ». Tout sert ce qui est étranger est qualifié de bizarre, primitif, étranger, irréaliste, etc. Mais le conformisme n'est souvent que superficiel, la majorité n'a pas de normes sur tout, et n'y tient pas toujours très fort. Elle n'a souvent d'unité que négative, comme indifférence et « inertie », dirait Sartre. L'enjeu, pour les minorités, ce sont les indécis, et non les adversaires déclarés, qu'il est vain de chercher à convaincre, et qu'il faut seulement s'attacher à empêcher de progresser. La minorité peut alors pousser de nouvelles normes, elle peut innover. Elle peut aussi chercher le conflit, dans ce but. En provoquant des tensions elle va fracturer l'unité de la majorité, qui peut n'être qu'apparente - mais cela, elle ne le saura vraiment qu'au moment de l'épreuve. Les progrès que fait une minorité active en matière d'influence sont d'abord invisibles, car c'est dans le domaine privé («transcendant ») qu'ils se manifestent en premier. Les membres de la majorité, isolés les uns des autres, « sériels », peuvent être convaincus par la minorités, mais pas au point d'afficher publiquement leur soutien par des actes, pour différentes raisons (ils sont déjà pris sur un autre front, sont épuisés par le labeur quotidien, dans une situation familiale délicate etc.). Le progrès peut donc n'être que sourd et souterrain. Les engagements concrets sont d'abord isolés. Ici c'est un individu qui décide d'installer du solaire sur sa maison, en trouvant quelques fausses excuses, pour éviter d'être pris " pour un écolo » etc. Les stratagèmes sont divers, pour s'engager concrètement sans afficher de soutien univoque pour " un parti », dont la synthèse n'est pas encore satisfaisante. Mais de proche en proche, la synthèse opère. Et puis un beau jour on découvre que la majorité a changé. D'où la devise de Guillaume d'Orange, que Moscovici cite comme illustrant la

13 Moscovici S., Op. Cit., p. 123 
maxime qui peut guider le comportement d'une «minorité active » : «il n'est pas nécessaire d'espérer pour entreprendre, ni de réussir pour persévérer $»^{14}$.

Ce rôle qui peut sembler ingrat explique que les minoritaires ne soient généralement pas aimés. Mais ils peuvent être admirés. Ils le sont d'autant plus qu'ils paient de leur personne. Ce qui est aimé et reconnu ce n'est pas leur personne, qui est plutôt crainte, car les minoritaires sont malgré tout un facteur de trouble, mais les normes et les valeurs qu'ils défendent. Car le trouble est parfois jugé nécessaire, par la majorité, même si les individus qui la composent ne souhaitent pas s'engager en leur nom propre, pour diverses raisons au sein desquelles la lâcheté n'est pas toujours la première, contrairement à ce qu'on pourrait penser - ce peut être aussi les responsabilités familiales, les difficultés à dégager du temps, l'absence de consensus dans le couple sur la question traitée etc. "Quiconque découvre une vérité ou enfreint une loi injuste est salué pour avoir corrigé l'erreur ou pour s'être efforcé de nous délivrer de l'injustice. En même temps, existe un désir irrésistible de le réprimander pour avoir écarté une autre vérité ou pour avoir enfreint la loi. La pratique antique d'exécuter les messagers qui apportaient de mauvaises nouvelles n'était pas exempte d'un semblable désir de trouver un bouc émissaire. Le héros s'aperçoit aussi rapidement qu'on lui suppose certaines responsabilités. Peu après l'exécution de ses grands ennemis, la révolution se débarrasse de ses propres leaders. C'est précisément cela, la relation à double critère : d'une part, reconnaître et admirer le nouveau et l'exceptionnel, et, d'autre part, le désapprouver et le nier afin de réintégrer l'ordinaire et le normal $»^{15}$.

\section{Conclusion}

Pour ceux qui douteraient encore de l'intérêt de ces analyses, mentionnons que Moscovici est cité par Ernesto Laclau dans ses analyses sur le populisme ${ }^{16}$, dont on sait combien elles ont marqué l'analyse, à gauche, au travers notamment d'échanges avec Slavoj Zizek et Judith Butler ${ }^{17}$. Laclau s'appuie aussi sur Sartre et sa théorie des ensembles pratiques, rarement mobilisée dans les analyses des mouvements sociaux. Il s'agit d'une théorie qui ne s'inscrit ni dans une perspective purement culturaliste de « reconnaissance » ni dans une lecture « économique » des rapports humains, elle permet donc d'une certaine manière de les dépasser. Elle met le « faire » au cœur des stratégies, sans donner de priorité au « faire » économique ni au « faire » des partis politiques ou d'un gouvernement. Tous les «faires» de toute la société sont requis pour atteindre l'harmonie collective, nul ne peut entièrement attendre que les solutions viennent « d'ailleurs ». Ce n'est pas non plus une théorie simplement « anarchiste », qui se refuserait à prendre le pouvoir parce que ce serait conforter l'existence d'un Etat dont on voudrait se débarrasser. Si l'Etat n'est pas central, c'est parce qu'il ne regroupe qu'un faible pourcentage des forces matérielles qui sont à l'œuvre dans le monde, et ne peut prétendre les harmoniser à lui seul.

Avec son concept « d'anti-pouvoir $»^{18}$, Holloway a eu l'intuition (et encore, sans sortir de la seule lutte des classes) de ce que Sartre avait sans doute démontré : que le pouvoir n'est pas seulement dans le quasisouverain, agissant sur fond de séries établies (les « classes ») mais aussi dans les groupes, seuls porteurs des séries à venir. Ainsi seulement peut-on demander non pas de socialiser le nucléaire (ou le capitalisme), mais

14 Ibid., p. 60

15 Ibid., p. 235

16 Laclau E., La raison populiste, Paris, Seuil, 2008.

17 Butler J., Laclau E. \& S. Zizek, Contingency, hegemony, universality - contemporary dialogues on the Left, Verso, 2000.

18 Holloway J., Changer le monde sans prendre le pouvoir - le sens de la révolution aujourd'hui, Syllepse, 2008, Ed. Orig. 2005. 
de l'abolir ou d'en changer radicalement le contenu. Ce cadre théorique exige donc une pensée latérale, complémentaire, minoritaire, dans laquelle différents engagements sont nécessaires pour concourir à un même but, et cela tout simplement parce que les individus et les situations sont multiples et ne peuvent donc pas être considérées comme unifiées et identiques. L'unité à faire, et non donnée. Le moment dialectique est pleinement pris en compte. Pour atteindre le même but, on peut voter un jour pour les Verts, un autre pour le NPA, un troisième pour le Centre, selon la configuration de la situation. Même chose pour tous les engagements. Créer des espaces autonomes, se soustrayant à l'emprise des séries, sur lesquelles règnent éventuellement des quasi-souverains qui empêchent les gens de former des groupes, n'est pas une stratégie accessoire : c'est un élément vital, nécessaire de tout changement «social». A côté de la lutte pour le contrôle des séries (dont fait partie la lutte des classes), il y a la lutte pour l'orientation des séries, leur fusion, leur réorganisation, leur réorientation. Et pas seulement à la fin de l'Histoire, quand le Capital aura accompli la Ruse de l'Histoire : ici et maintenant. 